\title{
Zollinger-Ellison Syndrome: A Rare Case of Chronic Diarrhea
}

\author{
Ali Aamar ${ }^{\mathrm{a}, \mathrm{d}}$, Kamraan Madhani ${ }^{\mathrm{a}}$, Hafeezulhassan Virk ${ }^{\mathrm{b}}$ \\ Zeeshan Butt ${ }^{\mathrm{c}}$
}

\begin{abstract}
Zollinger-Ellison syndrome (ZES) is caused by hypersecretion of gastrin from duodenal or pancreatic gastrinomas. We report a case of a 57-year-old female who presented with chronic diarrhea. CT abdomen showed multiple liver masses. Liver biopsy suggested metastatic well-differentiated neuroendocrine tumor. Serum gastrin level was markedly elevated. MRI abdomen, somatostatin receptor scintigraphy and endoscopic ultrasound failed to reveal primary site of the tumor. Upper gastrointestinal endoscopy showed hyperplastic gastric folds and multiple duodenal ulcers consistent with ZES. Patient was started on high-dose omeprazole and octreotide resulting in improvement in diarrhea.
\end{abstract}

Keywords: Zollinger-Ellison syndrome; Diarrhea; Gastrinoma

\section{Introduction}

Zollinger-Ellison syndrome (ZES) is caused by ectopic secretion of gastrin resulting in damage to gastrointestinal (GI) mucosa. ZES may be sporadic or occur in association with multiple endocrine neoplasia type 1 (MEN1). ZES is very rare and annual incidence is $0.5-2$ per million population [1]. ZES is usually diagnosed in fifth decade of life. In one-third of patients with gastrinoma, metastatic disease is evident at the time of diagnosis [2]. Diarrhea and abdominal pain are the most common presenting symptoms of ZES [3]. We report a case of ZES presenting with chronic diarrhea.

Manuscript accepted for publication December 08, 2016

anternal Medicine Residency Program, Yale-Waterbury, Waterbury, CT, USA

bInternal Medicine Residency Program, Mount Sinai St. Luke, New York, NY, USA

'Internal Medicine Residency Program, Prince George's Hospital, Cheverly, MD, USA

dCorresponding Author: Ali Aamar, Internal Medicine Residency Program, Yale-Waterbury, 64 Robbins Street, Waterbury, CT 06708, USA.

Email: ali.aamar@yale.edu

doi: https://doi.org/10.14740/gr734w

\section{Case Report}

A 57-year-old female presented with watery diarrhea for 10 months. She lost 70 pounds in this time period. On physical examination, she appeared cachectic. Abdomen was mildly distended with mild generalized tenderness. Laboratory analysis showed hemoglobin $11.5 \mathrm{~g} / \mathrm{dL}$, total protein $5.2 \mathrm{~g} / \mathrm{dL}$ and albumin $2.5 \mathrm{~g} / \mathrm{dL}$. Stool culture, ova, parasites and Clostridium difficile were negative. Fecal fat analysis revealed normal fat quantity. CT abdomen and PET scan showed multiple liver masses (Fig. 1A). Liver biopsy suggested metastatic welldifferentiated neuroendocrine tumor. Serum gastrin level was significantly elevated 9,100 pg/mL (normal $<100 \mathrm{pg} / \mathrm{mL}$ ). Serum vasoactive intestinal polypeptide and urine 5-hydroxyindoleacetic acid levels were normal. Serum chromogranin level was elevated. MRI abdomen, somatostatin receptor scintigraphy and endoscopic ultrasound failed to reveal primary site of neuroendocrine tumor. Colonoscopy was normal. Esophagogastroduodenoscopy showed prominent gastric folds and multiple duodenal ulcers (Fig. 1B) consistent with ZES. Patient was started on high-dose omeprazole and octreotide. Diarrhea improved and serum gastrin level normalized within 4 weeks.

\section{Discussion}

ZES is a clinical syndrome caused by hypersecretion of gastrin by a gastrinoma. Gastrinomas are located in the duodenum $(60-80 \%)$ or pancreas $(10-40 \%)$ [4]. Most of gastrinomas $(80 \%)$ are sporadic but $20-30 \%$ occur in association with MEN1. Although gastrinomas are one of the most common pancreatic neuroendocrine tumors (NETs), only 30\% arise in pancreas [5]. Abdominal pain (75\%) and diarrhea (73\%) are the most common clinical features of ZES. Diarrhea is caused by gastric acid hypersecretion and decreased absorption of sodium and water due to hypergastrinemia. Patients can also have heartburn (44\%) and weight loss (17\%). GI bleeding can be initial presentation in $25 \%$ of the patients. Renal stones are more common in patients with MEN1 compared to sporadic cases [6].

ZES should be considered in patients with diarrhea and peptic ulcer disease. Patients diagnosed with ZES should also be evaluated for MEN1. Fasting serum gastrin levels more 


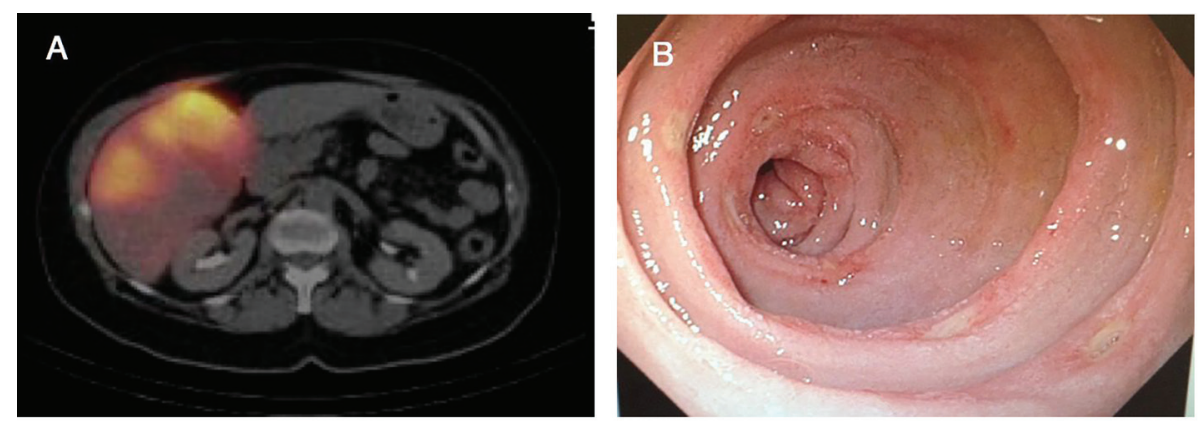

Figure 1. (A) PET scan showing liver metastases. (B) Upper GI endoscopy showing multiple duodenal ulcers.

than 10 times the upper limit of normal $(>1,000 \mathrm{pg} / \mathrm{mL})$ in the presence of low gastric $\mathrm{pH}(<2)$ is diagnostic of ZES. Secondary hypergastrinemia can be seen in atrophic gastritis, renal failure and proton-pump inhibitor (PPI) use [7]. More than $90 \%$ of the patients with ZES have peptic ulcers and hyperplastic gastric folds [3]. Once diagnosis of ZES is established, gastrinoma can be identified with a CT, MRI or somatostatin receptor scintigraphy [8].

Medical therapy with high-dose PPIs is the standard of care for ZES patients with MEN1 syndrome. Patients with ZES should be started on high-dose PPIs (omeprazole $60 \mathrm{mg}$ or pantoprazole $120 \mathrm{mg}$ daily). PPIs effectively control the symptoms in most of the patients with ZES. If symptoms persist despite of PPI use, somatostatin analogues should be used [9]. Patients with a sporadic gastrinoma and without evidence of metastases can be treated with surgery. Hepatic resection is indicated for patients with focal hepatic metastases. Hepatic artery embolization can be performed to control symptoms in patients with diffuse liver metastases [10].

\section{Funding Sources}

None.

\section{Competing Interests}

None.

\section{Author Contributions}

All authors have directly participated in the execution in a manner substantial enough to take responsibility for it.

\section{References}

1. Oberg K. Pancreatic endocrine tumors. Semin Oncol.
2010;37(6):594-618.

2. Sinagra E, Perricone G, Linea C, Montalbano L, Plano S, Simonetti RG, Orlando A, et al. An unusual presentation of zollinger-ellison syndrome. Case Rep Gastroenterol. 2013;7(1):1-6.

3. Roy PK, Venzon DJ, Shojamanesh H, Abou-Saif A, Peghini P, Doppman JL, Gibril F, et al. Zollinger-Ellison syndrome. Clinical presentation in 261 patients. Medicine (Baltimore). 2000;79(6):379-411.

4. Jensen RT, Niederle B, Mitry E, Ramage JK, Steinmuller $\mathrm{T}$, Lewington V, Scarpa A, et al. Gastrinoma (duodenal and pancreatic). Neuroendocrinology. 2006;84(3):173182.

5. Isenberg JI, Walsh JH, Grossman MI. Zollinger-Ellison syndrome. Gastroenterology. 1973;65(1):140-165.

6. Hoffmann KM, Gibril F, Entsuah LK, Serrano J, Jensen RT. Patients with multiple endocrine neoplasia type 1 with gastrinomas have an increased risk of severe esophageal disease including stricture and the premalignant condition, Barrett's esophagus. J Clin Endocrinol Metab. 2006;91(1):204-212.

7. Berna MJ, Hoffmann KM, Serrano J, Gibril F, Jensen RT. Serum gastrin in Zollinger-Ellison syndrome: I. Prospective study of fasting serum gastrin in 309 patients from the National Institutes of Health and comparison with 2229 cases from the literature. Medicine (Baltimore). 2006;85(6):295-330.

8. Metz DC, Jensen RT. Gastrointestinal neuroendocrine tumors: pancreatic endocrine tumors. Gastroenterology. 2008;135(5):1469-1492.

9. Metz DC, Comer GM, Soffer E, Forsmark CE, Cryer B, Chey W, Pisegna JR. Three-year oral pantoprazole administration is effective for patients with Zollinger-Ellison syndrome and other hypersecretory conditions. Aliment Pharmacol Ther. 2006;23(3):437-444.

10. Mayo SC, de Jong MC, Bloomston M, Pulitano C, Clary BM, Reddy SK, Clark Gamblin T, et al. Surgery versus intra-arterial therapy for neuroendocrine liver metastasis: a multicenter international analysis. Ann Surg Oncol. 2011;18(13):3657-3665. 